\title{
SOME PROPERTIES OF A GENERALIZED HAUSDORFF MEAN
}

\author{
C. W. LEININGER
}

1. Introduction. If $d$ is a moment sequence, i.e. $d_{n}=\int_{[0,1]} I^{n} d g$, $n=0,1,2, \cdots$, it is well known that the Hausdorff mean $H(d)$ is conservative if and only if there is a solution $g$ of the foregoing Hausdorff moment problem which is of bounded variation on $[0,1]$. Also well known are necessary and sufficient conditions for the existence of such a mass function $g$. The discovery by J. H. Wells [5] of necessary and sufficient conditions for the existence of a quasicontinuous solution of the Hausdorff moment problem, and the extension of these conditions by the author [4] to the case of a solution Riemannintegrable on $[0,1]$, have motivated the search for a generalization of the Hausdorff transformation which under certain conditions preserves convergence if the associated moment sequence is generated by a Riemann-integrable mass function.

In the classical theory of Hausdorff summability the mean $H(d)$ may be developed from the sequence-to-sequence transformation

$$
u_{n}=\Delta^{n} t_{0}=\sum_{p=0}^{n}(-1)^{p}\left(\begin{array}{l}
n \\
p
\end{array}\right) t_{p}
$$

We begin with the transformation

$$
u_{n}=\sum_{p=0}^{n}(-1)^{p}\left(\begin{array}{l}
n \\
p
\end{array}\right)_{s} t_{p}
$$

such that if $s$ is a sequence of positive numbers and $n, p$ is a nonnegative integer pair, then

$$
\left(\begin{array}{l}
n \\
p
\end{array}\right)
$$

denotes 0 if $n<p, 1$ if $n=p$, and $s_{n} \cdot s_{n-1} \cdots s_{p+1} /(n-p)$ ! if $n>p$. If $S$ denotes the matrix

$$
\left[(-1)^{p}\left(\begin{array}{l}
n \\
p
\end{array}\right)_{s}\right]
$$

Presented in part to the Society, April 20, 1968 under the title Concerning the inclusion problem for a generalized Hausdorff mean; received by the editors March 6 , 1967 and, in revised form, September 29, 1967. 
$d$ a number sequence and $D$ the diagonal matrix $\left[\delta_{n_{p}} d_{p}\right]$, and if $H^{(s)}=S^{-1} D S$, then $H^{(s)}$ is the matrix corresponding to the generalized Hausdorff mean which we denote by $H^{(s)}(d)$. It is readily seen that if $s_{n}=n, n=1,2,3, \cdots$, then $H^{(s)}(d)$ is $H(d)$. Parallel with the classical theory we find that $S^{-1}=S$,

$$
H_{n p}^{(s)}=\left(\begin{array}{l}
n \\
p
\end{array}\right)_{s} \Delta^{n-p} d_{p},
$$

if $b$ is a number sequence, the matrices corresponding to $H^{(s)}(d)$ and $H^{(s)}(b)$ commute, and if $X$ is a matrix commutable with the matrix of $H^{(s)}(d)$, then, provided $d_{n}=d_{m}$ implies $n=m$, there is a sequence $b$ such that $X$ is the matrix of $H^{(s)}(b)$. Furthermore, this generalization is essentially the only one with the above properties.

With $g$ restricted to a function of bounded variation on $[0,1],{ }^{1}$ conditions for convergence-preservation by $H^{(s)}(d)$ are established, an example of a nonconservative $H^{(s)}(d)$ mean is given, and the convergence domains of $H(d)$ and $H^{(s)}(d)$ are compared.

2. The generalized mean. The motivation for the generalization chosen is to retain the form

$$
u_{n}=\sum_{p=0}^{n}\left(\begin{array}{l}
n \\
p
\end{array}\right) \Delta^{n-p} d_{p} t_{p}
$$

of the Hausdorff transformation in the case $s_{n}=n, n=1,2,3, \cdots$.

THEOREM 1. $S^{-1}=S$ and for each nonnegative integer pair $n, p$,

$$
H_{n p}^{(s)}=\left(\begin{array}{l}
n \\
p
\end{array}\right)_{s} \Delta^{n-p} d_{p} .
$$
then

Proof. From the definitions, $S_{n n}^{2}=1$ and $S_{n p}=0$ if $n<p$. If $n>p$,

$$
\begin{aligned}
\sum_{k=0}^{n} S_{n k} S_{k p} & =\sum_{k=p}^{n}(-1)^{k}\left(\begin{array}{l}
n \\
k
\end{array}\right)_{s}(-1)^{p}\left(\begin{array}{l}
k \\
p
\end{array}\right)_{s} \\
& =\sum_{k=p}^{n}(-1)^{k+p} S_{n} \cdot s_{n-1} \cdots s_{p+1} /(n-k) !(k-p) ! \\
& =\sum_{k=p}^{n}(-1)^{k+p}\left(\begin{array}{l}
n-p \\
n-k
\end{array}\right)\left(\begin{array}{l}
n \\
p
\end{array}\right)_{s}=0 .
\end{aligned}
$$

1 The author wishes to thank the referee for suggesting this restriction in the present paper as well as for his other very helpful comments. 
Hence $S^{2}=I$.

If $n, p$ is a nonnegative integer pair, then

$$
\begin{aligned}
H_{n p}^{(s)} & =\sum_{k=0}^{n} S_{n k} d_{k} S_{k p} \\
& =\sum_{k=p}^{n}(-1)^{k+p}\left(\begin{array}{l}
n \\
p
\end{array}\right)_{s}\left(\begin{array}{l}
n-p \\
n-k
\end{array}\right) d_{k} \\
& =\left(\begin{array}{l}
n \\
p
\end{array}\right)_{s}^{n-p}(-1)^{k}\left(\begin{array}{c}
n-p \\
n-p-k
\end{array}\right) d_{p+k} \\
& =\left(\begin{array}{l}
n \\
p
\end{array}\right)_{s} \Delta^{n-p} d_{p} .
\end{aligned}
$$

We next observe that the $H^{(s)}(d)$ mean is essentially unique with respect to the desired properties.

THEOREM 2. Suppose (i) $G$ is a row-finite matrix of numbers such that $G^{-1}=G, G_{00}=1$, and if $n \geqq 1, G_{n 0}>0$ and $G_{n, n-1} \neq 0$.

(ii) $D$ is a diagonal matrix of numbers.

(iii) $A=G D G$.

(iv) For each nonnegative integer pair $n, p$ with $n \geqq p, A_{n p}$ $=\left|G_{n p}\right| \Delta^{n-p} d_{p}$.

Then if $s_{n}=\left|G_{n, n-1}\right|, n=1,2,3, \cdots, G=S$.

Proof. A simple computation shows that $G_{n n}=(-1)^{n}$ and $G_{20}$ $=-G_{10} G_{21} / 2>0$. Suppose $k$ is the least positive integer such that there is a positive integer $r, r<k$, such that

$$
G_{k r} \neq(-1)^{r}\left(\begin{array}{l}
k \\
r
\end{array}\right)_{s}
$$

Now

$$
A_{k 0}=\sum_{p=0}^{k} G_{k p} d_{p} G_{p 0}=G_{k 0} \Delta^{k} d_{0}=G_{k 0} \sum_{p=0}^{k}(-1)^{p}\left(\begin{array}{l}
k \\
p
\end{array}\right) d_{p} .
$$

Since $G_{k 0}>0$,

$$
G_{k r}\left(\begin{array}{l}
r \\
0
\end{array}\right)_{s}=G_{k 0}(-1)^{r}\left(\begin{array}{l}
k \\
r
\end{array}\right), \quad 0<r<k,
$$

or 


$$
\begin{aligned}
G_{k 0} & =\left|G_{k 1}\right| s_{1} /\left(\begin{array}{l}
k \\
1
\end{array}\right)=\left|G_{k 2}\right| s_{2} s_{1} / 2 !\left(\begin{array}{l}
k \\
2
\end{array}\right)=\cdots \\
& =\left|G_{k, k-2}\right| s_{k-2} s_{k-3} \cdots s_{1} /(k-2) !\left(\begin{array}{c}
k \\
k-2
\end{array}\right) \\
& =\left|G_{k, k-1}\right| s_{k-1} s_{k-2} \cdots s_{1} /(k-1) !\left(\begin{array}{c}
k \\
k-1
\end{array}\right) .
\end{aligned}
$$

But $\left|G_{k, k-1}\right|=s_{k}$, whence $\left|G_{k, k-2}\right|=s_{k} s_{k-1} / 2$ !, and it follows by induction that

$$
\left|G_{k r}\right|=\left(\begin{array}{l}
k \\
r
\end{array}\right)_{s}, \quad r=0,1, \cdots, k
$$

and

$$
G_{k r}=(-1)^{r}\left(\begin{array}{l}
k \\
r
\end{array}\right)_{s} .
$$

The next two theorems are stated without proofs as they may be established by methods analogous to those used in [3, Theorems 197, 198, p. 249].

TheOREm 3. If each of $b$ and $d$ is a number sequence, $B=\left[\delta_{n p} b_{p}\right]$, $X=S B S$ and $H^{(s)}=S D S$, then $H^{(s)} X=X H^{(s)}$.

THEOREM 4. If $d$ is a number sequence such that $d_{n}=d_{m}$ implies $n=m, H^{(s)}=S D S$ and $X$ is a matrix such that $H^{(s)} X=X H^{(s)}$, then there is a number sequence $b$ such that $X$ is the matrix of $H^{(s)}(b)$.

3. Convergence-preservation. Before considering more general moment sequences it seems advisable to investigate the $H^{(s)}(d)$ method in the context of the $H(d)$ method. Accordingly the moment sequences in this paper will be restricted to those generated by a function of bounded variation on $[0,1]$, and we denote by $B V$ the space of such sequences. Furthermore, the treatment will be considerably simplified by restricting $s$ so that $s_{n} \leqq n, n=1,2,3, \cdots$.

We observe that if $0<\alpha<1$ and $s_{n}=n-1+\alpha$, then $H^{(8)}(d)$ is essentially the $H^{(\alpha)}(\mu)$ method studied extensively by K. Endl [2] who, however, does not place this restriction on $\alpha$.

If $d \in B V$, it is apparent that with $s_{n} \leqq n, n \geqq 1$, an $H^{(s)}(d)$ mean satisfies two of the Silverman-Toeplitz conditions for convergencepreservation, so that we need consider only the sequence of the rowsums of $H^{(s)}$. If $p \geqq 0$, let $\pi^{(p)}$ denote the sequence such that $\pi_{p}^{(p)}=1$ 
and $\pi_{n}^{(p)}=\prod_{k=p+1}^{n} s_{k} / k, n>p$. With $\pi$ replacing $\pi^{(0)}$ and $\rho$ denoting the sequence $\left\{1 / \pi_{n}\right\}$, we have the following lemma, the routine proof of which is omitted.

Lemma. If $n \geqq 0$,

$$
\sum_{p=0}^{n} H_{n p}^{(s)}=\sum_{p=0}^{n} \pi_{n}^{(p)} H_{n p}=\pi_{n} \sum_{p=0}^{n} H_{n p} \rho_{p}=\pi_{n} \sum_{p=0}^{n}(-1)^{p}\left(\begin{array}{l}
n \\
p
\end{array}\right) \Delta^{p} \rho_{0} d_{p}
$$

and

$$
\sum_{p=0}^{n} H_{n p}=\rho_{n} \sum_{p=0}^{n} H_{n p}^{(s)} \pi_{p} .
$$

There is a fundamental theorem which follows directly from the lemma.

THEOREM 5. If $d \in B V$, an $H^{(s)}(d)$ mean is conservative if and only if the matrix

$$
\left[(-1)^{p} \pi_{n}\left(\begin{array}{l}
n \\
p
\end{array}\right) \Delta^{p} \rho_{0}\right]
$$

is conservative over the space $B V$.

The next three theorems give sufficient conditions for convergencepreservation. To facilitate comparison of the conditions, we introduce the sequence $a$ such that $a_{n}=1-s_{n} / n, n=1,2,3, \cdots$.

THEOREM 6. If $d \in B V$ and $\sum_{n} a_{n}$ is convergent, then $H^{(s)}(d)$ is conservative.

Proof. If $x$ is a convergent sequence and $l_{0}=\lim _{n} H_{n 0}$, then $\lim _{H} x$ $=\chi(H) \lim x+l_{0} x_{0}$ where $\chi(H)=d_{0}-l_{0}[6, \mathrm{p} .93]$. If $u=\lim \pi$, then $u>0$, so that $\lim \rho=1 / u$. Therefore, using the lemma,

$$
\lim _{n} \sum_{p=0}^{n} H_{n p}^{(s)}=u \lim _{n} \sum_{p=0}^{n} H_{n p} \rho_{p}=u\left[\left(d_{0}-l_{0}\right) / u+l_{0}\right]=d_{0}+l_{0}(u-1) .
$$

CoROLlaRy. If $d \in B V$ and $\sum_{n} a_{n}$ is convergent, then $H^{(s)}(d)$ is regular if and only if $H(d)$ is regular.

THEOREM 7. If $d \in B V$ and for each positive integer $n, a_{n+1} \geqq$ $n a_{n} /(n+1)$, then $H^{(s)}(d)$ is multiplicative.

Proof. Since $d$ is the difference of totally monotone sequences, it may be assumed that $d$ is totally monotone. From the lemma, 


$$
\begin{aligned}
\sum_{p=0}^{n} H_{n p}^{(s)}= & \pi_{n} \sum_{p=0}^{n} H_{n p} \rho_{p} \\
= & \pi_{n} \sum_{p=0}^{n} \rho_{p}\left(\begin{array}{l}
n \\
p
\end{array}\right)\left[H_{n+1, p} /\left(\begin{array}{c}
n+1 \\
p
\end{array}\right)+H_{n+1, p+1} /\left(\begin{array}{c}
n+1 \\
p+1
\end{array}\right)\right] \\
= & \pi_{n} H_{n+1,0}+\pi_{n} \sum_{p=1}^{n} H_{n+1, p}\left[\left(\begin{array}{c}
n \\
p-1
\end{array}\right) \rho_{p-1}+\left(\begin{array}{c}
n \\
p
\end{array}\right) \rho_{p}\right] /\left(\begin{array}{c}
n+1 \\
p
\end{array}\right) \\
& \quad+d_{n+1} \\
= & \pi_{n} H_{n+1,0}+\pi_{n} \sum_{p=1}^{n} H_{n+1, p}\left[p \rho_{p-1}+(n-p+1) \rho_{p}\right] /(n+1) \\
& \quad+d_{n+1} .
\end{aligned}
$$

If $1 \leqq p \leqq n$, then

$$
\begin{aligned}
\pi_{n} H_{n+1, p} & {\left[p \rho_{p-1}+(n-p+1) \rho_{p}\right] /(n+1)-H_{n+1, p}^{(s)} } \\
= & \pi_{n} H_{n+1, p} \rho_{p}\left\{\left[p \rho_{p-1} \pi_{p}+(n-p+1)\right] /(n+1)-s_{n+1} /(n+1)\right\} \\
= & \pi_{n} H_{n+1, p} \rho_{p}\left\{\left[p\left(1-a_{p}\right)+(n-p+1)\right] /(n+1)-\left(1-a_{n+1}\right)\right\} \\
= & \pi_{n} H_{n+1, p} \rho_{p}\left\{a_{n+1}-p a_{p} /(n+1)\right\} \geqq 0 .
\end{aligned}
$$

Therefore,

$$
\begin{aligned}
\sum_{p=0}^{n+1} H_{n+1, p}^{(s)} & =H_{n+1,0}^{(8)}+\sum_{p=1}^{n+1} H_{n+1, p}^{(8)} \\
& \leqq \sum_{p=0}^{n} H_{n p}^{(s)}-\left(\pi_{n}-\pi_{n+1}\right) H_{n+1,0} \\
& \leqq \sum_{p=0}^{n} H_{n p}^{(s)}
\end{aligned}
$$

since $H_{n+1,0} \geqq 0$ and $\pi_{n+1} \leqq \pi_{n}$. Furthermore, $\sum_{n} a_{n}$ is divergent so that $\lim \pi=0$.

It is interesting to note that if $0<\alpha<1$ and $s_{n}=n-1+\alpha, n \geqq 1$, then $a_{n}=(1-\alpha) / n$, and the $H^{(\alpha)}(\mu)$ method of Endl [2, pp. 426-429] satisfies the condition of the theorem with $a_{n+1}=n a_{n} /(n+1), n$ $=1,2,3, \cdots$.

THEOREM 8. If $d \in B V$ and for each positive integer $n, a_{n+1} \leqq$ $n a_{n} /(n+1)$, then $H^{(s)}(d)$ is conservative.

Proof. In view of Theorem 6 we may assume $\sum_{n} a_{n}$ to be divergent. Following the argument for Theorem 7 yields 


$$
\sum_{p=0}^{n+1} H_{n+1, p}^{(s)} \geqq \sum_{p=0}^{n} H_{n p}^{(s)}-\left(\pi_{n}-\pi_{n+1}\right) H_{n+1,0} .
$$

Hence if $k$ is a positive integer,

$$
\sum_{p=0}^{n+k} H_{n+k, p}^{(s)} \geqq \sum_{p=0}^{n} H_{n p}^{(s)}-\left(\pi_{n}-\pi_{n+k}\right) d_{0} .
$$

Since $\lim \pi=0$, if $\epsilon>0$, there is a positive integer $N_{1}$ such that if $n>N_{1}$, then

$$
\sum_{p=0}^{n+k} H_{n+k, p}^{(8)}>\sum_{p=0}^{n} H_{n p}^{(s)}-\epsilon .
$$

Since

$$
\sum_{p=0}^{n} H_{n p}^{(s)} \leqq d_{0}, \quad n \geqq 0,
$$

there is a positive integer $N, N \geqq N_{1}$, such that if $n>N$, then

$$
\sum_{p=0}^{n+k} H_{n+k, p}^{(8)}<\sum_{p=0}^{n} H_{n p}^{(s)}+\epsilon .
$$

For an example of a nonconservative $H^{(s)}(d)$ mean we take $H(d)$ to be the Cesàro mean of order one and let $a_{n}=3 / 4$ if $n=2^{2 k-1}, k$ $=1,2,3, \cdots$, with $a_{n}=0$ otherwise. Then if $n=2^{2 k-1}-1, \sum_{p=0}^{n} H_{n p}^{(s)}$ $\geqq 3 / 4$, while if $n=2^{2 k-1}, \sum_{p=0}^{n} H_{n p}^{(s)} \leqq 1 / 2$.

4. The inclusion problem. With $m$ denoting the space of bounded sequences, we consider the efficiency over $m$ of $H(d)$ and $H^{(s)}(d)$.

Theorem 9. If $d \in B V$ and $\lim \pi>0$, then $H^{(s)}(d)$ and $H(d)$ are equivalent over $n$.

Proof. Suppose $x \in m$ and $v=\lim _{H} x$. Then if it exists,

$$
\lim _{H^{(s)}} x=\lim _{n} \pi_{n} \sum_{p=0}^{n} H_{n p} \rho_{p} x_{p}=\lim \pi \lim _{n} \sum_{p=0}^{n} H_{n p} x_{p} \rho_{p} .
$$

If $u=\lim \pi$ and $l_{0}=\lim _{n} H_{n 0}$, then [6, p. 93]

$$
\begin{aligned}
\lim _{H^{(s)}} x & =u\left[\left(\lim _{H} x-\sum_{p} \lim _{n} H_{n p} x_{p}\right) \lim \rho+\sum_{p} \rho_{p} \lim _{n} H_{n p} x_{p}\right] \\
& =u\left[\left(v-l_{0} x_{0}\right) / u+\rho_{0} l_{0} x_{0}\right] \\
& =v-l_{0} x_{0}(1-u) .
\end{aligned}
$$


Suppose $w=\lim _{H}(s) x$. Then

$$
\begin{aligned}
\lim _{H} x & =\lim \rho \lim _{n} \sum_{p=0}^{n} H_{n p}^{(s)} x_{p} \pi_{p} \\
& =(1 / u)\left[\left(w-u l_{0} x_{0}\right) u+\pi_{0} u l_{0} x_{0}\right] \\
& =w-l_{0} x_{0}(u-1) .
\end{aligned}
$$

Since from the definition of $H^{(s)}(d)$ it is apparent that $\left[H^{(s)}\right]^{-1}=S D^{-1} S$, we may, for the case $\lim d \neq 0$, compare the relative strengths of an $H^{(s)}(d)$ mean and an $H(d)$ mean by using conditions on the inverse transformations.

TheOREM 10. If $d \in B V$ and $H^{(s)}(d)$ and $H^{-1}(d)$ are conservative, then $H^{(s)}(d)$ and $H(d)$ are equivalent.

Proof. If $x$ is a divergent sequence and $H x$ is convergent, then $H^{-1}(H x)$ is convergent. Hence $H(d)$ sums no divergent sequence. Since $\left\|H^{-1}\right\|$ exists, $\left\|\left[H^{(s)}\right]^{-1}\right\|$ exists, and it is well known (e.g., see $\left[6\right.$, p. 232]) that $\left[H^{(s)}\right]^{-1}(d)$ is conservative, so that $H^{(s)}(d)$ sums no divergent sequence.

The foregoing argument also establishes the following theorem.

Theorem 11. If $d \in B V$ and $H^{(s)}(d)$ and $\left[H^{(s)}\right]^{-1}(d)$ are conservative, then $H(d)$ includes $H^{(s)}(d)$.

Finally, the case in which $H^{(s)}(d)$ is conservative and neither $H^{-1}(d)$ nor $\left[H^{(s)}\right]^{-1}(d)$ is conservative remains an open question, some aspects of which are dealt with in the following comments.

Suppose $d \in B V, \lim d=0$ and $H(d)$ is regular. Then there is a bounded sequence which is not $H(d)$-summable. If $s_{n} / n=1 / 2^{n}, n$ $=1,2,3, \cdots$, then $\lim _{n} \sum_{p=0}^{n}\left|H_{n p}(s)\right|=0$, so that $H^{(s)}(d)$ sums every bounded sequence and thus includes $H(d)$. Let $\sigma$ denote the set of divergent sequences such that $t \in \sigma$ if and only if $t_{i}=0$ or $1, i=0,1$, $2, \cdots$. If $H(d)$ is a Cesàro mean of order $r, r \geqq 1$, then almost all sequences of $\sigma$ are $H(d)$-summable to $1 / 2$ [1, pp. 211-212]. If $\sum_{n} a_{n}$ is divergent and $x$ is a convergent sequence, there is a number $w$ such that $\lim _{H}(s) x=w x$, and it follows from $[1$, p. 211, 8.6, IV] that almost all sequences of $\sigma$ are $H^{(s)}(d)$-summable to $w / 2$. On the other hand, if $\lim d \neq 0$ and $H(d)$ is multiplicative, then neither $H(d)$ nor $H^{(s)}(d)$ sums to the same limit each of the sequences of a subset of $\sigma$ which is of positive measure.

\section{REFERENCES}

1. R. G. Cooke, Infinite matrices and sequence spaces, Macmillan, London, 1950. 
2. K. Endl, Untersuchungen über Momentenprobleme bei Verfahren vom Hausdorfschen Typus, Math. Ann. 139 (1960), 403-432.

3. G. H. Hardy, Divergent series, Clarendon Press, Oxford, 1956.

4. C. W. Leininger, A note on the Hausdorff moment problem, Proc. Amer. Math. Soc. 16 (1965), 1146-1147.

5. J. H. Wells, Concerning the Hausdorff inclusion problem, Duke Math. J. 26 (1959), 629-645.

6. Albert Wilansky, Functional analysis, Blaisdell, New York, 1964.

UNIVERSITY OF DALlas 